\title{
Dietary fish oil reduces skeletal muscle oxygen consumption, provides fatigue resistance and improves contractile recovery in the rat in vivo hindlimb
}

\author{
Gregory E. Peoples ${ }^{1 *}$ and Peter L. McLennan ${ }^{2}$ \\ ${ }^{1}$ School of Health Sciences and Smart Foods Centre, University of Wollongong, Wollongong, 2522 NSW, Australia \\ ${ }^{2}$ Graduate School of Medicine, University of Wollongong, Wollongong, 2522 NSW, Australia
}

(Received 2 February 2010 - Revised 25 May 2010 - Accepted 23 June 2010 - First published online 9 August 2010)

Dietary fish oil modulates skeletal muscle membrane fatty acid composition. Similar changes in heart membrane composition modulate myocardial oxygen consumption and enhance mechanical performance. The rat in vivo autologous perfused hindlimb was used to investigate the influence of membrane composition on skeletal muscle function. Male Wistar rats were fed either saturated fat (SF), $n$-6 PUFA (linoleic acid rich) or $n$-3 PUFA (fish oil) diets for 8 weeks. Hindlimb skeletal muscle perfused using the animal's own blood was stimulated via the sciatic nerve $(1 \mathrm{~Hz}, 6-12 \mathrm{~V}, 0.05 \mathrm{~ms})$ to contract in repeated $10 \mathrm{~min}$ bouts. The $n-3$ PUFA diet markedly increased $22: 6 n-3$ DHA, total $n-3$ PUFA and decreased the $n-6: n-3$ PUFA ratio $(P<0 \cdot 05)$ in red and white skeletal muscle membranes. There was no difference in initial twitch tension but the $n$-3 PUFA group maintained greater twitch tension within all contraction bouts and recovered better during rest to produce greater twitch tension throughout the final contraction bout $(P<0.05)$. Hindlimb oxygen consumption during contraction was significantly lower in the $n-3$ PUFA group compared with the SF group, producing a significantly higher $\mathrm{O}_{2}$ efficiency index compared with both SF and $n-6$ PUFA groups $(P<0.05)$. Resting oxygen consumption was increased in recovery in the SF group $(P<0.05)$ but did not change in the $n$-3 PUFA group. Membrane incorporation of $n$-3 PUFA DHA following fish oil feeding was associated with increased efficiency of muscle $\mathrm{O}_{2}$ consumption and promoted resistance to muscle fatigue.

Fish oil: $n$-3 PUFA: Oxygen consumption: Muscle fatigue: DHA

Dietary fatty acids affect structural membrane lipid composition, which in turn is strongly linked to cellular function ${ }^{(1)}$, including myocardial cell function ${ }^{(2)}$. Although tissue fatty acid composition reflects the types of fatty acids in the $\operatorname{diet}^{(3-6)}$, patterns of incorporation vary according to the tissue. In particular, heart myocardial membranes incorporate the $n$ - 3 PUFA DHA, from the diet, but do so in concentrations well above the circulating concentrations ${ }^{(6)}$. Although less studied, skeletal muscle appears to share this property of membrane sensitivity to incorporation of DHA with fish oil diet intervention ${ }^{(6-8)}$. Furthermore, skeletal muscle membrane composition is influenced by exercise training $^{(9-11)}$. In skeletal muscle, the incorporation of membrane $n$-3 PUFA may even be fibre-type specific and possibly be related to the high oxidative capacity of type I and IIa muscle cells where high levels of PUFA have been measured $^{(12,13)}$.

There is extensive evidence that incorporation of dietary $n-3$ PUFA into myocardial membranes has cellular ${ }^{(2)}$ and direct effects on heart function. Fish oil feeding to increase dietary $n-3$ PUFA has been demonstrated to enhance mechanical performance of the heart in the marmoset monkey ${ }^{(14,15)}$ and rat $^{(16,17)}$, decrease heart rate in exercising horses ${ }^{(18)}$ and in rats ${ }^{(5)}$, stabilise membranes to reduce arrhythmia vulnerability in rats ${ }^{(4)}$ and marmosets ${ }^{(19)}$ and modulate myocardial $\mathrm{O}_{2}$ consumption in rats ${ }^{(17)}$. In humans, the direct effects of dietary fish oil in the myocardium include reduced fatal arrhythmia ${ }^{(20)}$ and reduced resting ${ }^{(21,22)}$ and exercising heart rate ${ }^{(23)}$. The small but consistent reduction in heart rate has also been distilled from many clinical trials through meta-analysis ${ }^{(24)}$ and indirectly supports the notion that myocardial $\mathrm{O}_{2}$ consumption is reduced when fish is in the diet. Furthermore, there is evidence for reduction in whole body $\mathrm{O}_{2}$ consumption in well-trained cyclists following dietary fish oil intervention ${ }^{(23)}$, perhaps reflecting lower $\mathrm{O}_{2}$ consumption by the working skeletal muscle. Reduced $\mathrm{O}_{2}$ demand might produce fatigue resistance but study of isolated muscle has produced contradictory results and none have measured $\mathrm{O}_{2}$ consumption. In view of limitations in $\mathrm{O}_{2}$ delivery to isolated muscle bundles, an autologous, blood-perfused hindlimb method was developed $^{(25)}$ to investigate the effects of dietary $n-3$ PUFA on the skeletal muscle $\mathrm{O}_{2}$ consumption and contractile function, measuring both short-term fatigue during repeated contractions and twitch contractile recovery between contraction bouts. The premise of the present study was the confirmed changes in skeletal muscle membrane fatty acid composition through dietary intervention would directly modify skeletal muscle $\mathrm{O}_{2}$ consumption parallel to changes previously

Abbreviation: SF, saturated fat.

* Corresponding author: Dr G. E. Peoples, fax +61 24221 3486, email peoples@uow.edu.au 
reported for heart, which may be expressed as resistance to muscle fatigue.

\section{Methods}

Eighteen male Wistar rats $(400-500 \mathrm{~g})$ were used for the study (six per dietary intervention). Experiments were assessed and approved by the Animal Ethics Committee from the University of Wollongong, and all national and institutional guidelines were followed. Animals were housed two per cage in the University of Wollongong's animal facility with a room temperature maintained at $23-25^{\circ} \mathrm{C}$ and a $12 \mathrm{~h}$ light-dark cycle.

\section{Diet composition}

Three diets were prepared for the present study: saturated fat (SF); $n$-6 PUFA; $n$-3 PUFA. The diets all contained $10 \%$ fat by weight. The proportions of fat sources were selected to deliver similar total PUFA in the $n-6$ PUFA and $n-3$ PUFA diets, similar $n-6$ PUFA in the $n-3$ PUFA and SF diets and similar $n-3$ PUFA in the $n-6$ PUFA and SF diets. The final composition of each diet was established using GC against the known standards (Table 1). Olive oil was provided as light (refined) olive oil, therefore free of confounding sources of natural antioxidants; SF as beef tallow; $n-6$ PUFA as safflower oil; $n-3$ PUFA as high-DHA tuna fish oil $(28.9 \%$

Table 1. Fat sources and concentration of major fatty acids of experimental diets ( $\mathrm{g} / \mathrm{kg}$ diet $)^{*}$

\begin{tabular}{|c|c|c|c|}
\hline & \multicolumn{3}{|c|}{ Diet } \\
\hline & SF & $n-6$ PUFA & $n$-3 PUFA \\
\hline \multicolumn{4}{|l|}{ Fat source } \\
\hline Olive oil & 30 & 50 & 30 \\
\hline Beef tallow & 70 & & \\
\hline Safflower oil & & 50 & \\
\hline Fish oil & & & 70 \\
\hline \multicolumn{4}{|l|}{ Fatty acid } \\
\hline $14: 0$ & 2.59 & ND & $2 \cdot 12$ \\
\hline 16:0 Palmitic acid & 20.56 & 8.08 & 17.06 \\
\hline 16:1 Palmitoleic acid & $3 \cdot 16$ & 0.40 & 3.38 \\
\hline 18:0 Stearic acid & 14.08 & 3.63 & 4.50 \\
\hline 18: 1 Oleic acid & 47.94 & 49.97 & 33.52 \\
\hline 18: $2(n-6)$ Linoleic acid & 4.67 & $36 \cdot 00$ & 3.50 \\
\hline $18: 3(n-3) \alpha$-Linolenic acid & 0.58 & 0.49 & 0.56 \\
\hline $20: 4(n-6)$ Arachidonic acid & 0.04 & 0.06 & 1.30 \\
\hline $20: 5(n-3)$ EPA & ND & ND & 4.87 \\
\hline $22: 5(n-3)$ & ND & ND & 0.77 \\
\hline $22: 6(n-3) \mathrm{DHA}$ & ND & ND & $20 \cdot 20$ \\
\hline $\mathrm{EPA}+\mathrm{DHA}$ & 0 & 0 & $25 \cdot 07$ \\
\hline Total SFA & 38.06 & 12.59 & 25.48 \\
\hline Total MUFA & 51.39 & 50.59 & $39 \cdot 15$ \\
\hline Total PUFA & 5.34 & $36 \cdot 65$ & 31.40 \\
\hline$n-6$ PUFA & 4.77 & $36 \cdot 16$ & 5.01 \\
\hline$n-3$ PUFA & 0.58 & 0.49 & $26 \cdot 39$ \\
\hline$n-6: n-3$ ratio & 8.27 & 73.80 & 0.19 \\
\hline $\mathrm{P} / \mathrm{S}$ ratio & 0.14 & 2.91 & 1.23 \\
\hline UI & $62 \cdot 84$ & $124 \cdot 71$ & $203 \cdot 10$ \\
\hline
\end{tabular}

SF, saturated fat; P/S ratio, ratio of PUFA/SFA; $n-6: n-3$ ratio, ratio of $n-6$ PUFA to $n$-3 PUFA; UI, unsaturated index; ND, not detectable.

${ }^{*}$ Fatty acid concentrations as determined by GC.
DHA and 9\% EPA) (Clover Corporation, Altona North, VIC, Australia). Diets were prepared from purified ingredients and stored at $-20^{\circ} \mathrm{C}^{(26)}$.

\section{Study design}

Animals were fed a wash-out diet containing olive oil for $14 \mathrm{~d}$ to standardise muscle membrane fatty acid composition. They were then randomly allocated into three groups and fed ad libitum the SF, safflower oil (n-6 PUFA) or the tuna fish oil (n-3 PUFA) diet for 8 weeks. Fresh food was provided twice per week and daily consumption (g) was estimated by weighing the remainder from the previous feeding. Animal weight was measured once a week.

\section{Surgical preparation for ventilation, muscle perfusion and stimulation}

The autologous pump-perfused hindlimb has been previously described in both dogs ${ }^{(27)}$ and rats ${ }^{(25)}$. In brief, rats $(18-20$ weeks) were anaesthetised (sodium pentobarbital $6 \mathrm{mg} / 100 \mathrm{~g}$ intraperitoneally) with supplementary injections $(2 \mathrm{mg} / 100$ intraperitoneally) as required. Experiments were performed with the rat and perfusion system enclosed in a heated perspex chamber $\left(32.2 \pm 0.3^{\circ} \mathrm{C}\right)$ with animal body temperature maintained at $37^{\circ} \mathrm{C}$ (rectal temperature). The rats were prepared for artificial ventilation and blood pressure recording by cannulation of the trachea and left carotid artery, respectively. Rats were ventilated ( $1 \mathrm{ml} / 100 \mathrm{~g}$ body weight) to ensure constant high arterial $\mathrm{O}_{2}$ (Rodent Ventilator 7025, Ugo Basile, Italy).

All cannulae were fluid filled with saline containing $6 \%$ dextran (w/v) and $5000 \mathrm{IU}$ heparin/100 ml (Sigma-Aldrich, Castle Hill, NSW, Australia). Arterial (oxygenated) blood was accessed via a cannula inserted into the right femoral artery (non-perfused leg) towards the heart and was connected to a re-sealing flexi-tube, passed through a peristaltic roller pump (Gilson Minipuls 3, Middleton, WI, USA). Arterial blood was then passed through a cannula that was inserted into the left femoral artery (perfused leg) and positioned to deliver blood directly to muscle groups below the knee. A T-junction inserted in the blood flow line on the perfused hindlimb side of the pump was connected to a pressure transducer (Argon CDXIII; Maxim Medical, Athens, TX, USA) for measurement of hindlimb perfusion pressure. Venous blood (de-oxygenated) return to the heart and lungs was achieved via cannula inserted into the left femoral vein of the perfused leg and right jugular vein at the other end for passive flow.

Hindlimb muscle contraction was achieved via sciatic nerve stimulation. A bipolar electrode (F-B5EI; Grass Instrument Division, West Warwick, RI, USA) was placed under the nerves for direct electrical stimulation of the trunk supplying the muscles of the gastrocnemius-plantaris-soleus muscle bundle. The nerve was crushed above the stimulation point to prevent retrograde conduction. The gastrocnemiusplantaris-soleus muscle group tendons were tied with noncompliant silk and connected to a force transducer (FT03C; Grass Instrument Division). Saline-soaked gauze was placed over the muscles to prevent them from drying. 


\section{Hindlimb perfusion and stimulation protocol}

All hindlimbs were perfused for $30 \mathrm{~min}$ at $1 \mathrm{ml} / \mathrm{min}$ to allow for perfusion pressure to reach steady state (approximately $100 \mathrm{mmHg}$ ). Baseline arterial and venous blood samples $(400 \mu \mathrm{l})$ were drawn after $30 \mathrm{~min}$ of the initial perfusion (flow $=1 \mathrm{ml} / \mathrm{min}$ ) with no stimulation. Three separate $10 \mathrm{~min}$ stimulation bouts $(1 \mathrm{~Hz}, 7-12 \mathrm{~V}, 0.05 \mathrm{~ms})$ were delivered to the sciatic nerve separated by $30 \mathrm{~min}$ of muscle recovery. The flow rate was $1.5 \mathrm{ml} / \mathrm{min}$ during contraction periods and returned to $1 \mathrm{ml} / \mathrm{min}$ during the recovery periods. Arterial and venous samples were collected between the ninth and tenth minute of each contraction bout and between 25 and $30 \mathrm{~min}$ of recovery between bouts.

\section{Measurements}

\section{Blood pressure and twitch force}

Data were referenced to ground and amplified (Onspot Australia, Barrack Heights, NSW, Australia). The data acquisition software, Labview for Windows (National Instruments, Austin, TX, USA), was used to collect both pressure and twitch force simultaneously during the perfusion trials at a sampling rate of $200 \mathrm{~Hz}$.

\section{Blood samples}

Arterial and venous blood samples $(400 \mu \mathrm{l})$ were collected via the re-sealing silicone sections of the cannulae located proximal and distal to the hindlimb. Of the $400 \mu \mathrm{l}$ blood sampled, $240 \mu \mathrm{l}$ was presented to the laboratory blood gas and electrolyte machine (BGElectrolytes; Instrumentation Laboratory, Lexington, MA, USA) for measurement of $\mathrm{PO}_{2}$, $\mathrm{PCO}_{2}, \mathrm{Na}^{+}, \mathrm{K}^{+}, \mathrm{pH}$ and $\mathrm{Hb}$. The remaining 150-200 $\mu \mathrm{l}$ was spun down on a bench-top micro centrifuge (Milipore, North Ryde, NSW, Australia), the plasma was removed and frozen for later analysis. Erythrocytes from the plasma collections were re-suspended in an equal volume of saline and re-injected into the venous side of the perfusion. $\mathrm{Hb}$ levels were maintained above $12.5 \mathrm{~g} / 100 \mathrm{ml}$ whole blood.

\section{Muscle samples}

Control muscle samples (gastrocnemius, plantaris and soleus) were taken from the right (non-perfused) leg immediately before the right femoral artery cannulation, to be used as within-animal control tissue. At the completion of each experiment and while the blood was still flowing, left leg (perfused and contracted) muscle samples (gastrocnemius, plantaris and soleus) were rapidly extracted. All muscles were freezeclamped and stored $\left(-80^{\circ} \mathrm{C}\right)$ for later analysis. Soleus was used for red/oxidative tissue and gastrocnemius portion was used for white/glycolytic tissue. Additional muscles (deep posterior tibial, extensor digitorum longus and tibialis anterior) from the perfused hindlimb were collected to establish total weight of the muscle involved in the perfusion.

\section{Skeletal muscle membrane fatty acid analysis}

The method of membrane fatty acid analysis has been described previously ${ }^{(7)}$. In brief, $100-150 \mathrm{mg}$ of skeletal muscle tissue were weighed and homogenised in a chloroform-methanol mixture (2:1, v/v) (analytical grade Fluka; Sigma-Aldrich). Steps were taken for total lipid extraction using $1 \mathrm{M}-\mathrm{H}_{2} \mathrm{SO}_{4}$ (Sigma-Aldrich), phospholipid separation via silica seppack columns (Waters, Sutton, MA, USA), methylation of phospholipid fatty acids using $14 \%$ boron trifluoride in methanol ((Sigma-Aldrich) stored at $0-4^{\circ} \mathrm{C}$ ) heated at $85^{\circ} \mathrm{C}$ for $1 \mathrm{~h}$, purification via florisil Seppack columns (Waters) using diethyl ether (5\%) (Fluka; Sigma-Aldrich) in petroleum spirit (7 ml) (Fluka; Sigma-Aldrich) and finally GC (Shimadzu GC-17A, $30 \mathrm{~m} \times 0.25 \mathrm{~mm}$ internal diameter capillary column, total run time $23 \mathrm{~min}$ ). All solvents were freshly prepared at the time of analysis and contained $0.01 \%(\mathrm{w} / \mathrm{v})$ butylated hydroxy toluene (Sigma-Aldrich). Individual fatty acids were identified by comparison with the known standards in the laboratory. The relative amount of each fatty acid was determined by integrating the area under the peak and dividing by the result for all fatty acids detected. Unsaturation index, which represents the number of double bonds per hundred fatty acid molecules, was calculated by summing the products of the proportion (mol\%) of each fatty acid multiplied by the number of double bonds.

\section{Statistical analysis}

Computer software, Statistix, version 8 (Tallahassee, FL, USA), was used to analyse the data. One- (diet) and twoway (diet and time) ANOVA with individual means were compared using a corrected Bonferronni's post hoc analysis to indicate the significant difference. $\alpha$ was set at $P<0.05$ Data were expressed as means with their standard errors.

\section{Results}

\section{Food consumption and body weight}

There was no significant difference in the quantity of food consumed between dietary groups at either the start, the end or as a mean weekly intake over the 8 -week feeding period (SF: 6.85 (SEM 0.12); n-6 PUFA: 6.68 (SEM 0.15); $n$-3 PUFA: 6.70 (SEM 0.11) g/100 g body weight per week). Mean body weight was lower in the $n-6$ PUFA group (SF: 491 (SEM 27); $n$-6 PUFA: 427 (SEM 9); $n$-3 PUFA: 479 (SEM 15) g $(P<0 \cdot 05 n$-6) PUFA $v$. SF and $n$-3 PUFA). Despite the differences in mean body weight, there were no significant differences between dietary groups in mean total hindlimb muscle (SF: 4.48 (SEM 0.14); ( $n$-6) PUFA: 4.23 (SEM 0.17) n-3 PUFA: $4 \cdot 36$ (SEM 0.15) g wet weight) or gastrocneniusplataris-soleus muscle bundle (SF: 2.42 (SEM 0.19); n-6 PUFA: $2 \cdot 34$ (SEM 0.11); (n-3) PUFA: 2.20 (SEM 0.11) g wet weight).

\section{Skeletal muscle membrane fatty acids}

The fish oil fed group showed several marked membrane fatty acid concentration differences compared with the SF and $n-6$ PUFA groups, which did not differ from each other in any significant way (Table 2). In red and white portions of skeletal muscle, the $n$-3 PUFA group had significantly lower $18: 2 n-6 \mathrm{c}$ (linoleic acid), 20:4n-6 (arachidonic acid) and total n-6 PUFA $(P<0 \cdot 05)$ and significantly higher $20: 5 n-3$ (EPA), $22: 6 n-3$ 
Table 2. Skeletal muscle (red and white) membrane phospholipid fatty acid profiles ( $n 6)^{\star}$ (Mean values with their standard errors)

\begin{tabular}{|c|c|c|c|c|c|c|c|c|c|c|c|c|}
\hline & \multicolumn{4}{|c|}{ SF } & \multicolumn{4}{|c|}{$n-6$ PUFA } & \multicolumn{3}{|c|}{$n$-3 PUFA } & \\
\hline & \multicolumn{2}{|c|}{ Red } & \multicolumn{2}{|c|}{ White } & \multicolumn{2}{|c|}{ Red } & \multicolumn{2}{|c|}{ White } & \multicolumn{2}{|c|}{ Red } & \multicolumn{2}{|c|}{ White } \\
\hline & Mean & SEM & Mean & SEM & Mean & SEM & Mean & SEM & Mean & SEM & Mean & SEM \\
\hline $14: 0$ & 0.25 & 0.03 & 0.26 & 0.02 & 0.16 & 0.04 & 0.18 & 0.02 & 0.27 & 0.04 & 0.24 & 0.01 \\
\hline $16: 0$ & $17 \cdot 38$ & 3.76 & 20.63 & 0.72 & $18 \cdot 69$ & 1.95 & $21 \cdot 15$ & 0.52 & $19 \cdot 17$ & $4 \cdot 21$ & $22 \cdot 97$ & 0.83 \\
\hline $18: 0$ & 11.54 & $2 \cdot 34$ & 11.93 & 0.36 & 13.44 & 1.57 & $12 \cdot 10$ & 0.49 & $12 \cdot 82$ & 0.68 & $11 \cdot 21$ & 0.45 \\
\hline $18: 1 n-9$ & 8.64 & $2 \cdot 17$ & 9.60 & 1.25 & 8.86 & 1.35 & 7.42 & 0.78 & 8.29 & 0.55 & $7 \cdot 26$ & 0.32 \\
\hline $18: 2 n-6 c$ & $13.75^{\mathrm{b}}$ & 3.04 & $14.59^{\mathrm{b}}$ & 0.32 & $14.80^{b}$ & 1.58 & $15 \cdot 06^{\mathrm{b}}$ & 0.19 & $10.06^{a}$ & 0.67 & $9.63^{a}$ & 0.7 \\
\hline $20: 4 n-6$ & $15 \cdot 30^{\mathrm{b}}$ & 0.86 & $16 \cdot 63^{\mathrm{b}}$ & 0.68 & $17 \cdot 24^{b}$ & 2.29 & $20 \cdot 81^{b}$ & 1.47 & $10 \cdot 37^{\mathrm{a}}$ & 0.960 & $9.43^{\mathrm{a}}$ & 0.84 \\
\hline $20: 5 n-3$ & $0.11^{\mathrm{b}}$ & 0.07 & $0.04^{b}$ & 0.03 & nd & & nd & & $1.03^{\mathrm{a}}$ & 0.06 & $1 \cdot 11^{\mathrm{a}}$ & 0.07 \\
\hline $22: 5 n-3$ & 1.68 & 0.51 & 2.47 & 0.30 & 1.47 & 0.22 & 1.77 & 0.14 & $1 \cdot 18$ & 0.14 & 1.08 & 0.08 \\
\hline $22: 6 n-3$ & $13 \cdot 03^{b}$ & 3.36 & $9 \cdot 98^{\mathrm{b}}$ & 1.05 & $8.93^{b}$ & 0.98 & $9 \cdot 10^{\mathrm{b}}$ & 0.31 & $24 \cdot 36^{a}$ & 0.68 & $25 \cdot 16^{a}$ & 1.06 \\
\hline$\Sigma S F A$ & $29 \cdot 17$ & 6.0 & $32 \cdot 81$ & 1.0 & $32 \cdot 30$ & 3.42 & 33.43 & 0.95 & $36 \cdot 26$ & 1.29 & 34.42 & $1 \cdot 13$ \\
\hline ¿PUFA & 43.90 & 2.58 & $44 \cdot 26$ & 1.82 & $45 \cdot 63$ & 1.79 & $45 \cdot 30$ & $3 \cdot 27$ & $47 \cdot 19$ & 1.57 & $46 \cdot 68$ & 3.47 \\
\hline$\Sigma(n-6)$ & $30 \cdot 10^{b}$ & 3.44 & $33.89^{b}$ & $1 \cdot 74$ & $35 \cdot 37^{\mathrm{b}}$ & 1.79 & $36 \cdot 67^{b}$ & 1.47 & $20 \cdot 06^{a}$ & $1 \cdot 13$ & $20.53^{\mathrm{a}}$ & 0.70 \\
\hline$\Sigma(n-3)$ & $14.84^{\mathrm{b}}$ & $3 \cdot 12$ & $12.50^{\mathrm{b}}$ & $1 \cdot 18$ & $10 \cdot 41^{b}$ & $1 \cdot 17$ & $10 \cdot 88^{b}$ & 0.36 & $26 \cdot 49^{a}$ & 0.75 & $27.47^{\mathrm{a}}$ & $1 \cdot 19$ \\
\hline$n-6: n-3$ & $2 \cdot 75^{\mathrm{b}}$ & 0.53 & $2.79^{b}$ & 0.24 & $3.69^{b}$ & 0.65 & $3 \cdot 38^{b}$ & 0.16 & $0.76^{a}$ & 0.04 & $0.75^{a}$ & 0.0 .4 \\
\hline UI & $187^{\mathrm{b}}$ & 12 & $186^{\mathrm{b}}$ & 10 & $178^{\mathrm{b}}$ & 9 & $186^{\mathrm{b}}$ & 6 & $224^{\mathrm{a}}$ & 7 & $232^{\mathrm{a}}$ & 8 \\
\hline
\end{tabular}

SF, saturated fat; $\Sigma$ SFA, sum of SFA; $\Sigma$ PUFA, sum of PUFA; $\Sigma(n-6)$, sum of $(n-6)$ PUFA; $\Sigma(n-3)$, sum of $(n-3)$ PUFA; UI, unsaturation index; $n-6: n-3$, ratio of $n-6$ PUFA to $n-3$ PUFA; nd, not detectable.

${ }^{a, b}$ Mean values within a row with unlike superscript letters were significantly different $(P<0.05)$.

${ }^{*}$ Fatty acid concentrations as determined by GC.

(DHA) (Fig. 1(A), $P<0 \cdot 05$ ), and total $n$-3 PUFA (Fig. 1(B), $P<0.05)$ compared with both the SF and $(n-6)$ PUFA groups. This was reflected by the significantly lower $n-6: n-3$ PUFA ratio for the $n-3$ PUFA muscles (Table 2, $P<0 \cdot 05$ ). There were no significant differences that could be attributed to the red (soleus) or white (gastrocnemius) portion of muscle for these diets. The unsaturation index was significantly elevated in $n-3$ PUFA muscles compared with the SF and $n$-6 PUFA groups, which were not different from each other. There were no significant differences between groups in either total SFA or total PUFA.

\section{Baseline perfusion}

There was no significant difference in rectal temperature between dietary groups monitored during the procedure (SF: 37.0 (SEM 0.9); $n$-6 PUFA: 36.7 (SEM 0.6); $n$-3 PUFA: $36.0 \pm 0.7^{\circ} \mathrm{C}$ ). Hindlimb perfusion pressure after $30 \mathrm{~min}$ $(1 \mathrm{ml} / \mathrm{min})$ was significantly higher in the SF group compared with the $n$-3 PUFA group (SF: 126 (SEM 10); $n$-6 PUFA: 102 (SEM 14); $n$-3 PUFA: 93 (SEM 10) $\mathrm{mmHg})(P<0.05)$. There were no significant differences between dietary groups in the baseline arterial blood samples (Table 3). In all groups, artificial ventilation and resulting $\mathrm{PaO}_{2}$ were sufficient to achieve approximately $98 \% \mathrm{O}_{2}$ saturation of the erythrocytes and corresponding high content of arterial $\mathrm{O}_{2}$ supply (approximately $19-20 \mathrm{ml} / 100 \mathrm{ml}$ ). Baseline venous samples demonstrated a significant drop in $\mathrm{PO}_{2}$ compared with arterial blood $(P<0.05)$ and uptake of $\mathrm{O}_{2}$ across the resting muscle bundle. Although NS, the $n$-3 PUFA group demonstrated a trend for lower $(a-v) \mathrm{O}_{2}$ difference $(\mathrm{ml} / 100$ per $\mathrm{ml})$ and basal $\mathrm{O}_{2}$ consumption ( $\mu \mathrm{mol} / \mathrm{g}$ per min) (Table 3 ) compared with both the $n-6$ PUFA $(P=0 \cdot 13)$ and SF $(P=0.09)$ groups.
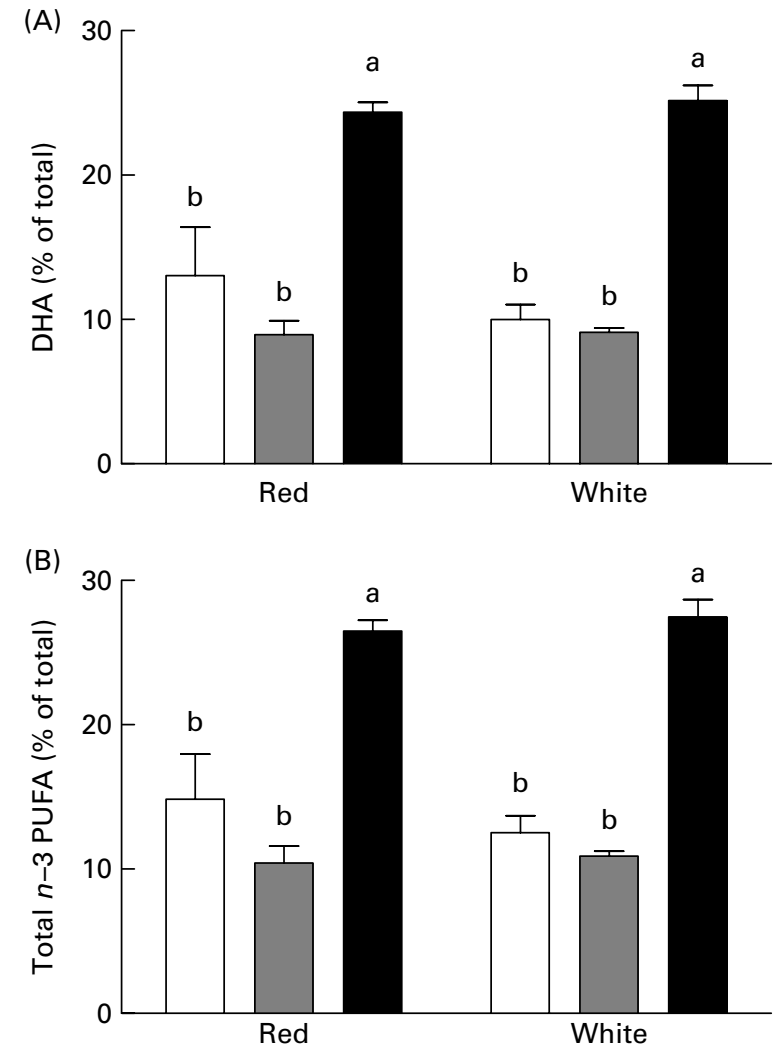

Fig. 1. Skeletal muscle PUFA phospholipid concentrations for saturated fat (SF), safflower oil ( $n-6$ PUFA) and DHA-rich fish oil (n-3 PUFA) after 8 weeks feeding. (A) Total $n-3$ PUFA; (B) DHA concentration. Mean values with unlike letters between diet groups were significantly different $(P<0.05)$. Mean values with their standard errors. $\square$, SF; $\square, n-6$ PUFA; 口, $n$-3 PUFA. 
Table 3. Baseline measurements of systemic arterial and venous hindlimb samples at a constant flow rate of $1 \mathrm{ml} / \mathrm{min}(n 6)$ (Mean values with their standard errors)

\begin{tabular}{|c|c|c|c|c|c|c|}
\hline & \multicolumn{2}{|c|}{ SF } & \multicolumn{2}{|c|}{$n-6$ PUFA } & \multicolumn{2}{|c|}{$n-3$ PUFA } \\
\hline & Mean & SEM & Mean & SEM & Mean & SEM \\
\hline \multicolumn{7}{|c|}{ Arterial blood gas and metabolite } \\
\hline $\mathrm{pH}$ & 7.53 & 0.03 & $7 \cdot 5$ & 0.01 & 7.55 & 0.02 \\
\hline $\mathrm{PaO}_{2}(\mathrm{mmHg})$ & 87 & 2 & 90 & 4 & 93 & 2 \\
\hline $\mathrm{PaCO}_{2}(\mathrm{mmHg})$ & 31 & 2 & 34 & 2 & 33 & 2 \\
\hline $\mathrm{Hb}(\mathrm{g} / 100 \mathrm{ml})$ & $13 \cdot 3$ & 0.3 & 13.9. & 0.5 & $14 \cdot 3$ & 0.5 \\
\hline $\mathrm{CaO}_{2}(\mathrm{ml} / 100 \mathrm{ml})$ & 18.96 & 0.7 & $19 \cdot 61$ & 0.5 & 19.93 & 0.8 \\
\hline $\mathrm{HCO}_{3}^{-}(\mathrm{mm})$ & $25 \cdot 8^{a}$ & $1 \cdot 1$ & $32 \cdot 1$ & 3.0 & $29 \cdot 2$ & $2 \cdot 3$ \\
\hline Lactate $(\mathrm{mm})$ & 2.92 & 0.17 & $2 \cdot 68$ & 0.12 & 2.76 & 0.24 \\
\hline \multicolumn{7}{|c|}{ Venous blood gas and metabolite } \\
\hline $\mathrm{pH}$ & 7.41 & 0.02 & 7.45 & 0.02 & 7.41 & 0.03 \\
\hline $\mathrm{PvO}_{2}(\mathrm{mmHg})$ & $40^{\mathrm{b}}$ & 2 & $43^{a, b}$ & 3 & $47^{\mathrm{a}}$ & 2 \\
\hline $\mathrm{PvCO}_{2}(\mathrm{mmHg})$ & 42 & 3 & 46 & 3 & 48 & 4 \\
\hline $\mathrm{Hb}(\mathrm{g} / 100 \mathrm{ml})$ & 13.75 & 0.5 & $14 \cdot 16$ & 0.4 & 14.4 & 0.5 \\
\hline $\mathrm{CvO}_{2}(\mathrm{ml} / 100 \mathrm{ml})$ & 14.38 & 1.0 & $15 \cdot 63$ & 0.7 & $16 \cdot 53$ & 0.9 \\
\hline $\mathrm{HCO}_{3}^{-}(\mathrm{mm})$ & $26 \cdot 2^{\mathrm{a}}$ & 1.2 & $32 \cdot 8^{\mathrm{b}}$ & $2 \cdot 8$ & $29 \cdot 9^{a, b}$ & 2.5 \\
\hline Lactate $(\mathrm{mm})$ & $2 \cdot 62$ & 0.2 & 3.66 & 0.19 & $3 \cdot 12$ & 0.5 \\
\hline \multicolumn{7}{|c|}{ Hindlimb resting oxygen consumption } \\
\hline$(a-v) \mathrm{O}_{2}(\mathrm{ml} / 100 \mathrm{ml})$ & 4.57 & 0.73 & 3.97 & 0.77 & 3.39 & 0.45 \\
\hline $\mathrm{VO}_{2}(\mu \mathrm{mol} / \mathrm{g}$ per $\mathrm{min})$ & 0.41 & 0.07 & 0.37 & 0.07 & 0.31 & 0.04 \\
\hline
\end{tabular}

SF, saturated fat.

${ }^{a, b}$ Mean values within a row with unlike superscript letters were significantly different $(P<0.05)$.

\section{Muscle contraction}

There was a significant decline in initial and maximum tensions between Bout 1 and Bout 3 independent of diet $(P<0.05)$ (Fig. 2). The decline was smallest in the $n$-3 PUFA group for both initial and maximum tension $(P=0.07$ and $0 \cdot 09$, respectively). There was no significant difference in the final tension between Bout 1 and Bout 3 independent of diet; however, final tension in the $n-3$ PUFA group was significantly higher compared with the SF group $(P<0.05)$. As hindlimb blood flow increased, perfusion pressure significantly increased on each occasion independent of dietary group $(P<0 \cdot 05)$. There were no significant effects of diet on hindlimb perfusion pressure during twitch contractions.

The recovery of initial, maximum and final twitch tension in Bout $3(1.5 \mathrm{ml} / \mathrm{min})$ was compared to Bout $1(1 \mathrm{ml} / \mathrm{min})$ (Fig. 3(A)). The $n-3$ PUFA group demonstrated a greater recovery of twitch tension in contraction Bout $3(P<0.05)$ compared with the SF and $n-6$ PUFA groups. Additionally, when final twitch tension was expressed as a percentage of maximum twitch tension (Fig. 3(B)), in bouts 1, 2 and 3, the $n$-3 PUFA group demonstrated greater maintenance of twitch tension over the $10 \mathrm{~min}(P<0 \cdot 05)$. Twitch characteristics including maximum rate of contraction $(+\mathrm{d} T / \mathrm{d} t)$ and maximum rate of relaxation $(-\mathrm{d} T / \mathrm{d} t)$ decreased across Bouts 1, 2 and 3 independent of diet. There was no significant difference between diets at the points of initial, maximum and final twitch tension (data not shown).

During contraction, the $(a-v) \mathrm{O}_{2}$ difference $(\mathrm{ml} / 100$ per $\mathrm{ml})$ and $\mathrm{O}_{2}$ consumption ( $\mu \mathrm{mol} / \mathrm{g}$ per min) (Fig. 4(B)) were significantly lower in the $n$-3 PUFA group compared with the SF group. The efficiency index, calculated as the twitch tension developed relative to muscle mass for a given amount of $\mathrm{O}_{2}$ consumed (N/g per $\mu$ mol per min), was significantly higher in the $n-3$ PUFA group across all exercise bouts compared with both SF and $n-6$ PUFA groups $(P<0 \cdot 05)($ Fig. $4(\mathrm{C}))$.

\section{Recovery between contraction bouts}

The hindlimb $\mathrm{O}_{2}$ consumption in the SF group was increased during recovery periods 1 and 2 compared with its own baseline (Fig. 4(A)) $(P<0.05)$. In comparison, $\mathrm{O}_{2}$ consumption returned towards original baseline levels in both the $n-3$ PUFA and $n-6$ PUFA groups. Notably, within each recovery period, $\mathrm{O}_{2}$ consumption for the $n-3$ PUFA group was significantly lower than that for the SF group $(P<0 \cdot 05)$.

\section{Discussion}

In rats fed fish oil, skeletal muscle was resistant to fatigue during continuous muscle twitch contractions and recovered contractile force better between repeat bouts. Importantly, the improved contractile performance in the fish oil-fed animals was achieved with reduced skeletal muscle $\mathrm{O}_{2}$ consumption both during contraction bouts and during recovery. These changes in muscle function were associated with the incorporation of $n-3$ PUFA into skeletal muscle membrane which reflected the well-documented membrane phospholipid responses to the dietary fish oil in excitable tissue ${ }^{(28)}$ including heart and skeletal muscle in the marmoset monkey ${ }^{(6)}, \operatorname{rat}^{(8)}$ and human subjects ${ }^{(29)}$.

\section{Skeletal muscle membrane fatty acid composition}

Large differences in skeletal muscle membrane fatty acid composition were achieved with 8 weeks of dietary manipulation; however, there was little indication in the present study that this was specific to muscle fibre type as previously suggested $^{(12,30)}$. There was some reduction in the concentration of SFA palmitic acid (16:0) of red fibres compared with that of the white fibres, which has been inversely related to insulin sensitivity ${ }^{(31)}$. In addition, when provision of essential fatty acids was low (SF feeding), the concentration of 

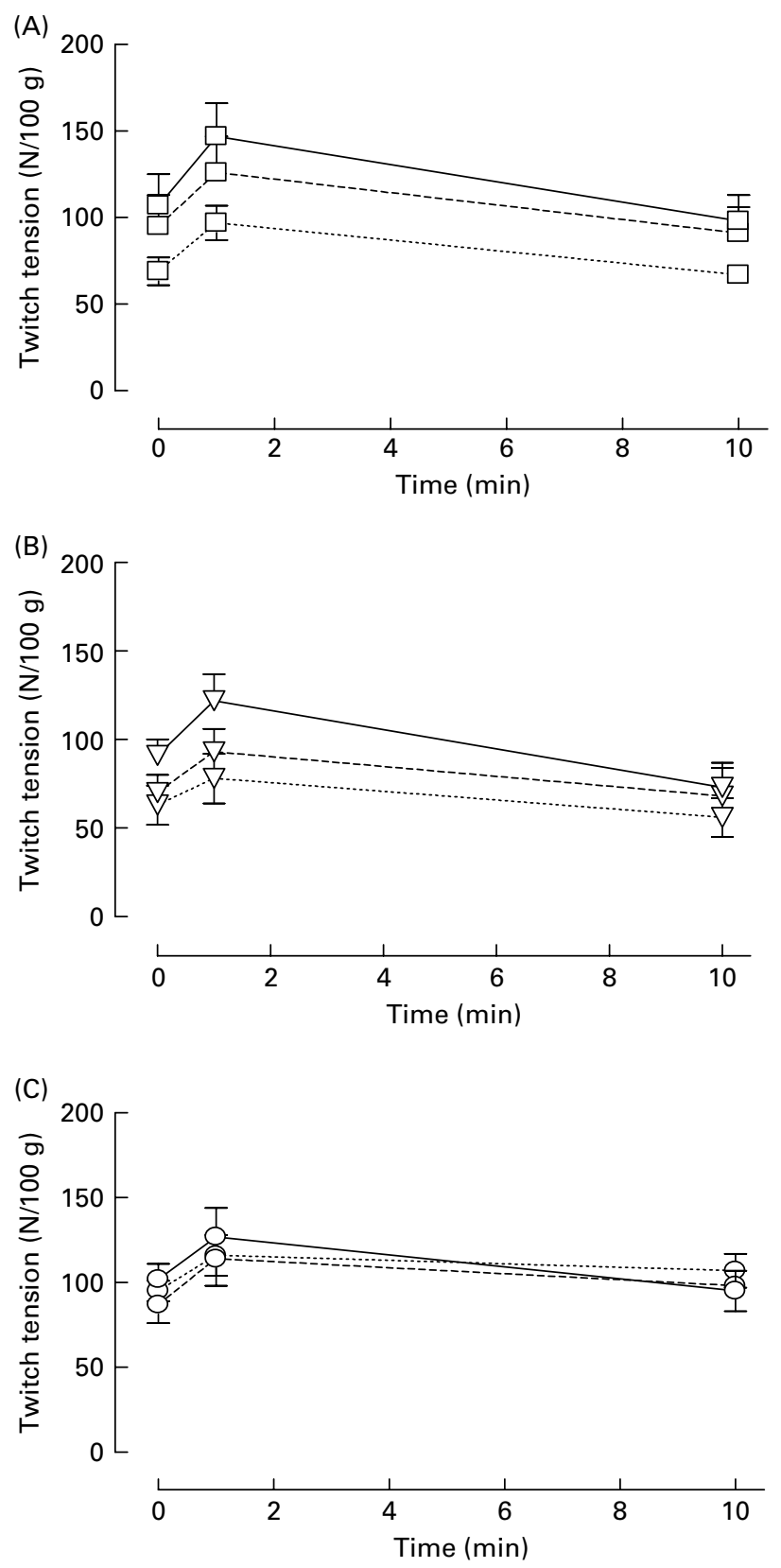

Fig. 2. Twitch tension development (N/g wet weight) for Bout 1 , Bout 2 and Bout 3 for (A) saturated fat (SF), $-\square-$, Bout $1 ;--\square--$, Bout $2 ; \cdots \square \cdots$, Bout 3 ; (B) $n-6$ PUFA (safflower oil), $-\nabla-$, Bout $1 ;--\nabla--$, Bout $2 ; \cdots \nabla \cdots$, Bout 3 and (C) $n$-3 PUFA (DHA-rich fish oil) groups, $-\mathrm{O}-$, Bout 1 ; --O--, Bout 2; $\cdots \circ \cdots$, Bout 3. Flow $=1.5 \mathrm{ml} / \mathrm{min}$. Initial tension (time $=0$ ), maximum tension (time $=60 \mathrm{~s}$ ) and final tension (time $=10 \mathrm{~min}$ ) are shown $(n 6)$. Values displayed as means with their standard errors.

DHA was retained at higher levels in the red muscle compared with that of the white muscle. That is, the high oxidative fibres retained higher DHA concentrations compared with the low oxidative fibres $^{(12)}$.

The long-chain $n-3$ PUFA DHA was the major fatty acid incorporated into skeletal muscle membranes with fish oil feeding, and the changes were comparable to those seen in the heart after similar dietary interventions ${ }^{(5,6,16)}$. Although the present study used a DHA-rich tuna fish oil supplement, it is evident that DHA incorporation is always the primary
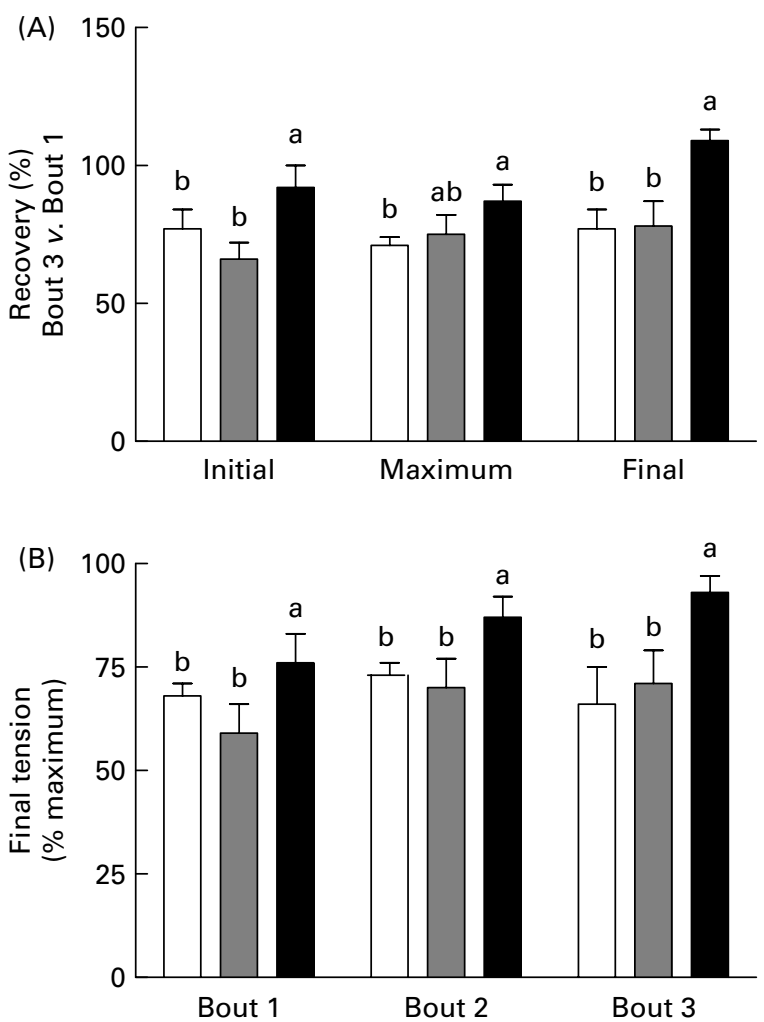

Fig. 3. (A) Recovery (\%) for initial, maximum and final twitsh tension Bout $3 v$. Bout 1. (B) Final twitch tension as a percentage of maximum twitch tension (Bout 1, Bout 2 and Bout 3). $\square$, SF; $\square, n$-6 PUFA; $\mathbf{n}, n$-3 PUFA. Mean values with unlike superscript letters between diet groups were significantly different $(P<0.05)(n 6)$. Values displayed as mean with their standard errors.

response of excitable tissue to long-chain $n$-3 PUFA fatty acids in the diet, even when EPA-rich fish oils or purified EPA are consumed ${ }^{(5,6)}$. A systematic comparison of tissue fatty acid composition of the marmoset monkey demonstrated remarkably similar fatty acid profiles between heart and skeletal muscle ${ }^{(6)}$, whereas other tissues such as vascular smooth muscle, platelets or kidney do not reflect the same extent of DHA incorporation. The DHA concentration in skeletal muscle of the marmoset monkey is very similar to the red and white skeletal muscle of the rat in the present study and further similarities were seen in both SF and n-6 PUFA groups, where DHA concentrations were lower and arachidonic acid concentrations were higher ${ }^{(6)}$. The similarity in fatty acids of heart and skeletal muscle merits the consideration that skeletal muscle could be used as an indirect marker of the heart $n$-3 PUFA composition ${ }^{(6)}$. It is already established that the fatty acid composition of human skeletal muscle reflects the diet ${ }^{(32)}$ and further use of the biopsy technique in skeletal muscle could allow for indirect monitoring of changes in the heart of human subjects.

\section{Skeletal muscle function}

This is the first study to investigate skeletal muscle function on the basis of the modulated $\mathrm{O}_{2}$ consumption previously demonstrated in the heart ${ }^{(17)}$, and its success is largely dependent on properties of the in vivo autologous hindlimb model ${ }^{(25)}$, adapted from canine muscle fatigue experiments ${ }^{(33)}$. Only a 
(A)
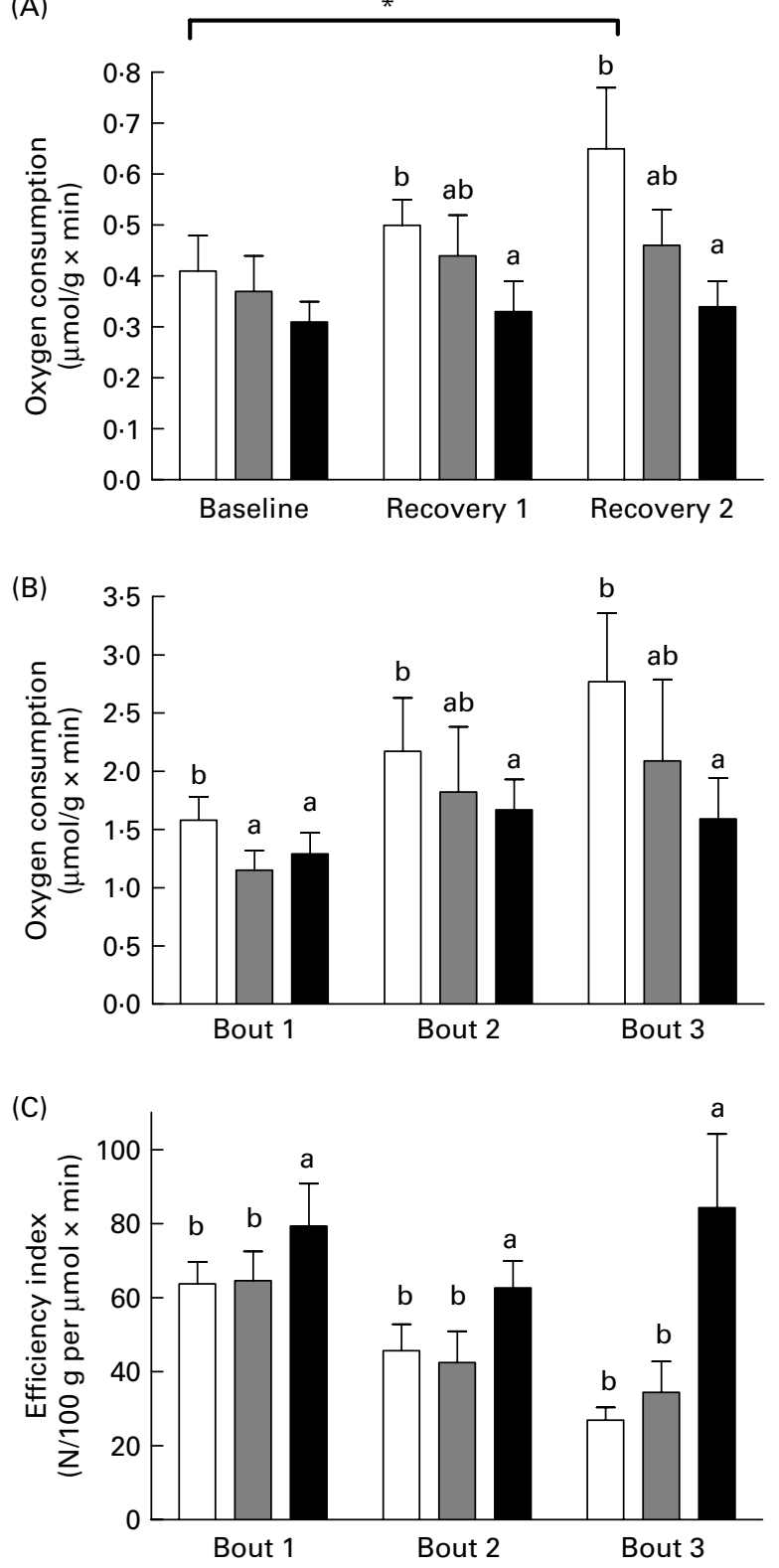

Fig. 4. (A) Oxygen consumption $(\mu \mathrm{mol} / \mathrm{g} \times \mathrm{min})$ at baseline and recovery 1 and recovery 2 (following Bout 1 and 2 respectively). Flow rate $=1 \mathrm{ml} / \mathrm{min}$, (B) contracting $\mathrm{O}_{2}$ consumption $(\mu \mathrm{mol} / \mathrm{g} \times \mathrm{min})$ and (C) efficiency index $(\mathrm{N} / 100 \mathrm{~g} \mathrm{per} \mu \mathrm{mol} \times \mathrm{min}$ ) at flow rate $1.5 \mathrm{ml} / \mathrm{min}$ (Bout 1, Bout 2 and Bout 3 ). $\square$, SF; $\square, n-6$ PUFA; $\mathbf{n}, n-3$ PUFA. Mean values with unlike superscript letters between diet groups were significantly different $(P<0.05)$. ${ }^{*}$ Mean values were significantly different within groups $(n 6) \quad(P<0.05)$. Values displayed as means with their standard errors.

few studies have considered the effects of dietary-induced changes in membrane composition on skeletal muscle function ${ }^{(8,34,35)}$. These studies used large muscle bundles in vitro, not reflective of in vivo $\mathrm{O}_{2}$ delivery $^{(36)}$ or voluntary exercise in vivo and produced contradictory outcomes.

The progressive fatigue of the gastrocnemius-plantarissoleus muscle bundle in vivo during repetitive twitch contractions was less evident in animals fed the $n-3$ PUFA diet, and fatigue resistance became even more apparent in repeat contraction bouts. Twitch force recovery between contraction bouts was also greater with $n$-3 PUFA feeding.
This is comparable to the enhanced cardiac contractile function $^{(14,15,37)}$ and improved post-ischaemic cardiac recovery ${ }^{(16,17)}$ seen in association with fish oil feeding.

The inherently low resting $\mathrm{O}_{2}$ consumption of perfused hindlimb was not affected by diet and concurs with findings from $\mathrm{O}_{2}$ consumption of the $\mathrm{K}^{+}$-arrested, quiescent heart ${ }^{(17)}$. In contrast, $\mathrm{O}_{2}$ consumption of the contracting hindlimb, evaluated during the final twitches of each contraction bout, increased in SF and $n-6$ PUFA hindlimbs, despite the decline in contractile function, but remained low in the $n-3$ PUFA group although muscle contraction was declining less. $\mathrm{O}_{2}$ consumption and efficiency were modulated when the muscle was put under conditions of increased $\mathrm{O}_{2}$ demand just as is observed in myocardium under stress such as increased filling pressure or acute myocardial ischaemia and reperfusion $^{(17)}$. This represents a clear increase in efficiency of muscle $\mathrm{O}_{2}$ use, again mimicking the effects observed in the heart ${ }^{(16)}$ where stress-induced increases in $\mathrm{O}_{2}$ use is moderated by fish oil feeding.

Like fish oil feeding in the present study, endurance training promotes greater recovery from bouts of exercise ${ }^{(38)}$. $\mathrm{O}_{2}$ consumption, $\mathrm{CO}_{2}$ production, minute ventilation and heart rate all show faster adjustment to relative and absolute exercise levels and recovery after exercise. Fish oil supplementation reduces whole body $\mathrm{O}_{2}$ consumption and heart rate throughout prolonged exercise bouts in trained cyclists ${ }^{(23)}$. The observations in the present study suggest that fish oil, like exercise training, may reduce fatigue and improve recovery after exercise.

The similarities between fish oil influences on skeletal muscle and myocardial composition and function suggest common underlying mechanisms. Identification of mechanisms of $n$-3 PUFA effects is more advanced for myocardium than for skeletal muscle. A vast array of $n$-3 PUFA-responsive cell signalling processes has been identified, including changes in eicosanoid and free-radical production ${ }^{(2)}$. Irrespective of the intracellular signalling, the $n-3$ PUFA are available through their prior incorporation into phospholipids of the cell membranes. Altered cellular $\mathrm{Ca}^{2+}$ handling is implicated in many of the effects of dietary fish oil in the heart ${ }^{(39)}$, including appearing as a common endpoint or intermediate for effects on myocardial intracellular signalling ${ }^{(2)}$. Excessive $\mathrm{O}_{2}$ consumption, cardiac arrhythmias and their modulation by $n-3$ PUFA have all been associated with conditions of cellular sarcoplasmic reticulum and mitochondrial $\mathrm{Ca}^{2+}$ overload $^{(17,40)}$. Although skeletal muscle does not mimic the Ca-induced Ca-release of myocardium, it still makes use of the basic properties of $\mathrm{Ca}$ release from the sarcoplasmic reticulum into the cytosol and re-uptake which is implicated in muscle fatigue ${ }^{(41)}$. Therefore, $\mathrm{Ca}^{2+}$ cycling processes appear as one potential mechanism of action of fish oil fatty acids in excitable cell function that may translate to skeletal muscle.

The higher unsaturation index of skeletal muscle from fish oil-fed rats implies an increased risk of cellular oxidative damage, which might translate into poor contractile performance in line with the free-radical theory of fatigue ${ }^{(42)}$. The paradoxical fatigue resistance provoked by fish oil feeding suggests that like ischaemic preconditioning ${ }^{(43)}$, and nutritional preconditioning by $n-3$ PUFA in the heart ${ }^{(16)}$, or exercise training ${ }^{(44)}$, the $n$-3 PUFA up-regulate the endogenous protective mechanisms in skeletal muscle. 
In summary, using the rat in vivo autologous pump-perfused hindlimb, fish oil feeding delayed muscle fatigue and improved contractile recovery in association with changes in membrane phospholipid fatty acid composition. As previously observed in heart, there is evidence for the modulation of skeletal muscle $\mathrm{O}_{2}$ consumption associated with increased membrane DHA levels. It is of primary interest to further investigate the levels of fatigue and the corresponding $\mathrm{O}_{2}$ consumption at the commencement of contraction and establish that these findings are reproducible at all points of isometric fatigue. Additionally, it is of interest to establish if these observations also hold true when the skeletal muscle is subjected to greater levels of contractile fatigue $(<50 \%$ maximum twitch tension) and longer duration of stimulation, which will also impact on the degree of fatigue. The present findings demonstrate the direct implications for dietary fats on skeletal muscle contractile performance and may have applications in cases of muscle fatigue and dysfunction such as heart failure and muscle dystrophy.

\section{Acknowledgements}

This project was supported by Australian Research Council Strategic Partnerships with Industry - Research and Training (ARC-SPIRT); Smart Foods Centre and Clover Corporation. The DHA-rich fish oil was provided by Clover Corporation. Each author contributed equally to the manuscript preparation. We declare that there are no conflicts of interest.

\section{References}

1. Gorjao R, Azevedo-Martins A, Rodrigues H, et al. (2009) Comparative effects of DHA and EPA on cell function. Pharmacol Therapeut 122, 56-64.

2. Siddiqui R, Harvey K \& Zaloga G (2008) Modulation of enzymatic activities by $n-3$ polyunsaturated fatty acids to support cardiovascular health. J Nutr Biochem 19, 417-437.

3. McLennan P, Howe P, Abeywardena M, et al. (1996) The cardiovascular protective role of docosahexaenoic acid. Eur J Pharmacol 300, 83-89.

4. McLennan P, Abeywardena MY \& Charnock JS (1988) Dietary fish oil prevents ventricular fibrillation following coronary artery occlusion and reperfusion. Am Heart J 116, 709-717.

5. Pepe S \& McLennan P (1996) Dietary fish oil confers direct antiarrhythmic properties on the myocardium of rats. $J$ Nutr 126, 34-42.

6. Charnock JS, Abeywardena MY, Poletti VM, et al. (1992) Differences in fatty acid composition of various tissues of the marmoset monkey after different lipids supplement diets. Comp Biochem Physiol 101A, 387-393.

7. Pan D \& Storlien LH (1993) Dietary lipid profile is a determinate of tissue phospholipids fatty acid composition and rate weight gain in rats. $J$ Nutr 123, 512-519.

8. Ayre K \& Hulbert A (1996) Dietary fatty acid profile influences the composition of skeletal muscle phospholipids in rats. $J$ Nutr 126, 653-662.

9. Helge JW, Ayre KJ, Hulbert AJ, et al. (1999) Regular exercise modulates muscle membrane phospholipid profile in rats. $J$ Nutr 129, $1636-1642$.

10. Helge JW, Wu BJ, Willer M, et al. (2001) Training affects muscle phospholipid fatty acid composition in humans. $J$ Appl Physiol 90, 670-677.
11. Andersson A, Sjodin A, Olsson R, et al. (1998) Effects of physical exercise on phospholipid fatty acid composition in skeletal muscle. Am J Physiol 274, E432-E438.

12. Kriketos AD, Pan DA, Sutton JR, et al. (1995) Relationship between membrane lipids, fibre type, and enzyme activities, in sedentary and exercise rats. Am J Physiol 269, R1154-R1162.

13. Pan DA, Lillioja S, Milner MR, et al. (1995) Skeletal muscle membrane lipid composition is related to adiposity and insulin action. J Clin Invest 96, 2802-2808.

14. McLennan PL, Barnden LR, Abeywardena MY, et al. (1989) Dietary polyunsaturated fatty acids improve cardiac function in the marmoset monkey. J Mol Cell Cardiol 21, 32.

15. Charnock JS, McLennan PL, McIntosh BR, et al. (1987) Radionuclide angio-graphic study of the influence of the dietary lipid supplements on the cardiac function in the marmoset (Callithrix jacchus). Cardiovasc Res 21, 369-376.

16. Abdukeyum G, Owen A \& McLennan P (2008) Dietary (n-3) long chain polyunsaturated fatty acids inhibit ischemia and reperfusion arrhythmias and infarction in rat heart not enhanced by ischemic preconditioning. J Nutr 138, 1902-1909.

17. Pepe S \& McLennan PL (2002) Cardiac membrane fatty acid composition modulates myocardial oxygen consumption and postischemic recovery of contractile function. Circulation 105, 2303-2308.

18. O'Connor C, Lawrence L, Lawrence A, et al. (2004) The effect of dietary fish oil supplementation on exercising horses. J Anim Sci 82, 2978-2984.

19. McLennan PL, Bridle TM \& Abeywardena M (1992) Dietary lipid modulation of ventricular fibrillation threshold in the marmoset monkey. Am Heart J 123, 1555-1561.

20. Burr ML, Gilbert JF, Holliday RM, et al. (1989) Effects of changes in fat, fish, and fibre intakes on death and myocardial reinfarction: diet and reinfarction trial (DART). Lancet 30, $757-761$.

21. Dallongeville J, Yarnell J, Ducimetiere P, et al. (2003) Fish consumption is associated with lower heart rates. Circulation 108, $820-825$.

22. Shah A, Ichiuji A, Han J, et al. (2007) Cardiovascular and endothelial effects of fish oil supplementation in health volunteers. J Cardiovasc Pharmacol Theraput 12, 213-219.

23. Peoples G, McLennan P, Howe P, et al. (2008) Fish oil reduces heart rate and oxygen consumption during exercise. J Cardiovasc Pharmacol 52, 540-547.

24. Mozaffarian D, Geelen A, Brouwer I, et al. (2005) Effect of fish oil on heart rate - A meta-analysis of randomised controlled trials. Circulation 112, 1945-1952.

25. Hoy A, Peoples G \& McLennan P (2009) The effect of vasoconstrictors on oxygen consumption in resting and contracting skeletal muscle of the autologous pump-perfused rate hindlimb. $J$ Physiol Pharmacol 60, 155-160.

26. Owen A, Peter-Przyborowska B, Hoy A, et al. (2004) Dietary fish oil dose- and time-response effects on cardiac phospholipid fatty acid composition. Lipids 39, 955-961.

27. Hogan MC, Gladden LB, Grassi B, et al. (1998) Bioenergetics of contracting skeletal muscle after partial reduction of blood flow. J Appl Physiol 84, 1882-1888.

28. Charnock JS (1982) Dietary lipids, cardiac performance, and enzyme function. Chemia 36, 249-250.

29. Storlien LH, Baur LA, Kriketos AD, et al. (1996) Dietary fats and insulin action. Diabetologia 39, 621-631.

30. Okano G, Matsuzaka H \& Shimojo T (1980) A comparative study of the lipid composition of white, intermediate, red and heart muscle in rats. Biochimica Biophys Acta 619, 167-175.

31. Vessby B, Tengblad S \& Lithell H (1994) Insulin sensitivity is realted to the fatty acid composition in serum lipids and skeletal muscle phospholipids in 70 year old men. Diabetologia 37, 1044-1050. 
32. Andersson A, Nalsen C, Tengblad S, et al. (2002) Fatty acid composition of skeletal muscle reflects dietary fat composition in humans. Am J Clin Nutr 76, 1222-1229.

33. Stainsby WN \& Welch HG (1966) Lactate metabolism of contracting dog skeletal muscle in situ. Am J Physiol 211, 177-183.

34. Ayre K \& Hulbert A (1996) Effects of changes in dietary fatty acids on isolated skeletal muscle function in rats. $J$ Appl Physiol 80, 464-471.

35. Ayre KJ \& Hulbert AJ (1997) Dietary fatty acid profile effects endurance in rats. Lipids 32, 1265-1270.

36. Zhang S, Bruton J, Katz A, et al. (2006) Limited oxygen diffusion accelerates fatigue development in mouse skeletal muscle. J Physiol 572, 551-559.

37. Demaison L, Blet J, Sergiel JP, et al. (2000) Effect of dietary polyunsaturated fatty acids on contractile function of hearts isolated from sedentary and trained rats. Reprod Nutr Dev 40, $113-125$.

38. Hagberg JM, Hickson RC, Eshani AA, et al. (1980) Faster adjustment to and recovery from submaximal exercise in the trained state. J Appl Physiol 48, 218-224.
39. McLennan P \& Abeywardena M (2005) Membrane basis for fish oil effects on the heart: linking natural hibernators to prevention of human sudden cardiac death. J Membr Biol 206, $85-102$.

40. Pepe S, Tsuchiya N, Lakatta EG, et al. (1999) PUFA and aging modulate cardiac mitochondrial membrane lipid composition and $\mathrm{Ca}^{2+}$ activation of PDH. Am J Physiol 276, H149-HH58.

41. Favero TG (1999) Sarcoplasmic reticulum $\mathrm{Ca}^{2+}$ release and muscle fatigue. J Appl Physiol 87, 471-483.

42. Ferreira L \& Reid M (2008) Muscle-derived ROS and thiol regulation in muscle fatigue. J Appl Physiol 104, 853-860.

43. al Makdessi S, Brandle M, Ehrt M, et al. (1995) Myocardial protection by ischemic preconditioning: the influence of the composition of myocardial phospholipids. Mol Cell Biochem 145, 69-73.

44. Pansarasa O, D'Antona G, Gualea M, et al. (2002) “'Oxidative stress": effects of mild endurance training and testosterone treatment on rat gastrocnemius muscle. Eur J Appl Physiol 87, $550-555$. 